\title{
Coffee or Tea? Examining Cross-Cultural Differences in Personality Nuances Across Former Colonies of the British Empire
}

\author{
Priscilla Achaa-Amankwaa ${ }^{1}$, Gabriel Olaru ${ }^{1}$, and Ulrich Schroeders ${ }^{1}$ \\ ${ }^{1}$ Department of Psychology, University of Kassel, Germany
}

\begin{abstract}
Author Note
Priscilla Achaa-Amankwaa (iD https://orcid.org/0000-0003-4087-5293

Gabriel Olaru (iD https://orcid.org/0000-0002-7430-7350

Ulrich Schroeders (iD https://orcid.org/0000-0002-5225-1122

We have no conflict of interest to disclose.

Correspondence concerning this article should be addressed to Priscilla Achaa-Amankwaa, Psychological Assessment, Institute of Psychology, University of Kassel, Holländische Str. 36-38, 34127 Kassel, Germany, Email: priscillaachaa@gmail.com
\end{abstract}

This is a preprint version of a manuscript submitted for publication (i.e., before peer-review). 


\title{
CROSS-CULTURAL DIFFERENCES IN PERSONALITY NUANCES
}

\begin{abstract}
Cross-cultural comparisons often focus on differences in broad personality traits across countries. However, many cross-cultural studies report differential item functioning which suggests that considerable group differences are not accounted for by the overarching personality factors. We argue that this may reflect cross-cultural personality differences at a lower level of personality, namely personality nuances. To investigate the degree of cultural similarities and differences between participants of ten countries that formerly belonged to the British Empire, we scrutinized participants’ personality scores on domain, facet, and nuance level of the personality hierarchy. More specifically, we used the responses of 9,110 participants on the IPIP-NEO 300-item personality inventory in cross-validated and regularized logistic regressions. Based on the trait domain and facet scores, we were able to identify the country of residence for $60 \%$ and $73 \%$ of the participants, respectively. By using the nuance level of personality, we correctly identified the nationality of $89 \%$ of the participants. This pattern of results explains the lack of measurement invariance in crosscultural studies. We discuss implications for cross-cultural personality research and whether the high degree of cross-cultural item-level differences compromises the universality of the personality structure.
\end{abstract}

Keywords: cross-cultural personality, personality nuances, machine learning 


\section{Coffee or Tea? Examining Cross-Cultural Differences in}

\section{Personality Nuances Across Former Colonies of the British Empire}

Personality is conceptualized as a higher-order construct in the most prominent models on the structure of personality such as the Five-Factor model (Costa \& McCrae, 1992; Goldberg, 1990) and the HEXACO model (Lee \& Ashton, 2004). At the top of the hierarchy are the domains (Neuroticism, Extraversion, Conscientiousness, Agreeableness, and Openness to Experience), which can be further divided into facets (e.g., Neuroticism encompasses the facets Anxiety, Vulnerability, Depression). More recent studies assume an even narrower level of personality below the facets, labeled personality nuances (McCrae, 2015; Mõttus et al., 2017). For instance, tension and worry can both be conceived as nuances of the Neuroticism facet Anxiety; although they are constructed to measure the same facet, they manifest differently either as behavior or emotion (McCrae, 2015). Several studies so far have demonstrated the predictive validity, heritability, and differential stability of personality nuances, often operationalized as single personality items (Mõttus et al., 2017; Mõttus \& Rozgonjuk, 2019; Seeboth \& Mõttus, 2018).

Research on cross-cultural differences in personality found small but substantial meanlevel differences in the personality trait domains and facets across countries (Allik et al., 2017; Allik \& McCrae, 2004; McCrae et al., 1998; McCrae \& Terracciano, 2005b; Schmitt et al., 2007). For example, it is found that European and North-American cultures are distinguishable to Asian and African cultures, with the former cultures appearing to be more outgoing, open to new experience, and antagonistic, while the latter cultures tend to be more introverted, traditional, and compliant (Allik et al., 2017; Allik \& McCrae, 2004). Accordingly, Extraversion seems to be much lower in Southern and Eastern regions of Asia as well as in South America than for example in North America, Europe, Africa, and Oceania (Schmitt et al., 2007). Hitherto, cross-cultural personality differences were only examined at the domain and facet level of personality. This approach assumes that the trait domains and 


\section{CROSS-CULTURAL DIFFERENCES IN PERSONALITY NUANCES}

facets account for the cultural differences observed at the nuance level of personality. Comparisons of the factor structure of the NEO-PI-R (Neuroticism Extraversion Openness Personality Inventory Revised; Costa \& McCrae, 1992) derived from principal component analysis suggested a universally applicable factor structure across countries (e.g., McCrae et al., 1998; McCrae, 2002; McCrae \& Terracciano, 2005a). However, more recent examinations of the factorial equivalence of personality across countries with stricter multi-group confirmatory factor analysis cast doubt on these findings: A systematic review (Dong \& Dumas, 2020) showed that only $43 \%$ of studies that tested for cross-cultural measurement invariance found support for metric measurement invariance (i.e., equal factor loadings), whereas full scalar measurement invariance (i.e., equivalent item intercepts across countries) has only been achieved in specifically designed short versions (Jankowsky et al., in press). These findings imply that the underlying personality factors do not fully account for the crosscultural differences present at the item or nuance level.

This lack of measurement invariance translates to the concept of differential item functioning (DIF) in an item response theory (Millsap, 2011). DIF occurs if respondents with different backgrounds (e.g., ethnic, age, or gender), but the same latent trait level, respond differently to an item (Clauser \& Mazor, 1998). In contrast to measurement invariance which operates on a scale level, DIF directs the focus of variation to the item level. However, items that function differently across cultures are not a nuisance to the researcher per se, but also allow insights into cultural personality differences. This situation has been aptly labeled the DIF paradox (Church et al., 2011). On one hand, researchers seek to exclude items that are non-invariant to draw unbiased comparisons at the facet and domain level of personality. On the other hand, the excluded items may convey important information in the understanding of cultural differences in personality. Consequently, the presence of DIF in itself is not 


\section{CROSS-CULTURAL DIFFERENCES IN PERSONALITY NUANCES}

necessarily problematic but can be of assistance in measuring personality-relevant cultural differences (Baer et al., 2011; Church et al., 2011).

In the present study, we tried to achieve two goals. First, we wanted to compare the degree of cultural differences at the trait domain, facet, and nuance level of personality by predicting the nationality of participants in a pairwise country comparison with scores of the respective level. We then used the classification accuracy as an estimate of the cross-cultural differences at the differnt levels in the personality hierarchy. Second, we tried to detecet clusters of cultural similarities in personality across 10 different countries. In the following, we discuss traditional perspectives on DIF in cross-cultural personality research, consider the implications of the nuance concept, and describe the procedures used in this study.

\section{Differential Item Functioning in Cross-Cultural Personality Research}

Huang et al. (1997) were one of the first to examine the cross-cultural measurementinvariance at the item level of a personality inventory such as the NEO-PI (McCrae \& Costa, 1985) and found about $40 \%$ of the English NEO-PI items to function differently across a Filipino and a U.S.-American sample. In the same vein, Church et al. (2011) examined the validity of cultural differences in personality profiles of the NEO-PI-R between the USA, Mexico, and the Philippines at item and facet level and reported that $40-50 \%$ of the items in the NEO-PI-R were affected to some extent by DIF between these three countries. The authors concluded that DIF was predominant in the NEO-PI-R and often carried forward to the facet level. In another study, more than a quarter of the 60 NEO-FFI items demonstrated non-invariant item loadings across Euro-American and Asian international groups (Rollock \& Lui, 2016). This is, however, not a specific shortcoming limited to the NEO-PI and its derivatives. Likewise, the items of the Multidimensional Personality Questionnaire (MPQ, Eigenhuis et al., 2013) showed (depending on the modeling concept) between 19-40\% DIF when comparing U.S.-American and Dutch samples (Eigenhuis et al., 2015). Such a lack of cross-cultural measurement invariance may point to measurement artifacts or some construct 


\section{CROSS-CULTURAL DIFFERENCES IN PERSONALITY NUANCES}

bias (e.g., behavior that is differentially appropriate). Several of those factors have been explored in the past and must be considered when comparing personality scores over countries and cultures (for a comprehensive overview see van de Vijver \& Tanzer, 2004). These include (a) translation errors, (b) response biases and differing self-presentation motives, (c) cultural specificity of the items, and (d) sampling errors, that restrict the representativity of the sample drawn for the respective culture. We will shortly give an example of each source of variance in the measurement.

A prominent example of translational issues has been reported by Davidov and colleagues (2014), who found that the incorrect translation of the word "crime" into Danish led to higher levels of tolerance towards criminal immigrants in the Danish sample compared to other countries. Several studies have shown that acquiescence levels, that is, the tendency to agree to statements regardless of their content, varied across cultures (Johnson et al., 2005; Wong et al., 2003). In a study conducted on European countries, Rammstedt and colleagues (2017) revealed that $15 \%$ of the variance in acquiescence in response styles could be linked to country-level variables such as corruption level and collectivism. Similarly, Allik and McCrae (2004) found Asian and Black African cultures to show less variation in personality scores than European cultures, which could be explained by the former cultures’ tendency to avoid extreme responses (Rollock \& Lui, 2016). The cultural specificity of certain items was reported by Church and colleagues (2011), who found that a NEO-PI-R Extraversion item measuring the tendency to go roller coasting produced strong DIF across the U.S. and Philippines, because of the lack of roller coasters in the latter country. Olaru and Danner (in press) found that the Big Five Inventory 2 (Soto \& John, 2017) Conscientiousness item "Is reliable, can always be counted on" was an indicator of Agreeableness for French and Spanish participants. Similarly, Safdar and colleagues (2009) showed that Extraversion items measuring positive emotions had considerable DIF between the U.S. and Japan, as openly 


\section{CROSS-CULTURAL DIFFERENCES IN PERSONALITY NUANCES}

showing emotions is discouraged in Japan. Instead, higher Extraversion levels were reflected in more assertive behavior in this country.

\section{Personality Nuances}

The crucial question is at what level of the personality hierarchy the aforementioned sources of measurement variance or cultural differences do manifest. Cross-cultural personality research has mostly focused on higher-order personality traits, providing an overview of geographic differences in personality on the domain and the facet level (e.g., Kajonius \& Mac Giolla, 2017; McCrae \& Terracciano, 2005b). This kind of research is confronted with questions of measurement invariance (Jankowsky et al., in press), sampling issues (Henrich et al., 2010), and especially the fact that the conceived personality models are inconsistent with the empirical data (Borsboom, 2006). The difficulties regarding the structure of personality are also reflected in the absence of agreement regarding the number of domains (Big 5 vs. HEXACO) or the facets below, and the degree of unidimensionality of the personality factors (e.g., circumplex models; Hofstee et al., 1992). As a result, alternative models of extracting the personality structure have been recently proposed such as network analysis (Epskamp, 2017) or exploratory structural equation modeling (Marsh et al., 2010). In this context, personality nuances have been introduced as a more fine-grained level of personality even below the facet level (McCrae, 2015; Mõttus, et al., 2017). In more detail, "the term nuance refers to different forms of a facet, corresponding operationally to individual items (or groups of conceptually redundant items)” (McCrae, 2015, p. 102). Items entail distinct unique personality-relevant variance, which is not accounted for by facets or domains (Mõttus et al., 2014). These personality nuances are suggested to be the most basic conceptual units of personality variance and the "building blocks” (Mõttus et al., 2018, p. 2-3) of the personality trait hierarchy. This conceptualization seems reasonable since the basic constituents of personality—feelings, thoughts, attitudes, motivation, and behavior—are most accurately and exhaustively captured at the item level, whereas some information is lost at 


\section{CROSS-CULTURAL DIFFERENCES IN PERSONALITY NUANCES}

higher levels of aggregation. In the present context, the question is to what extent the description of differences across countries is adequate on a facet or domain level, or whether personality nuances contribute substantially to the description and understanding of crosscultural differences in personality.

In some way, findings of DIF in personality measurement might be indicative of such unique item variance, contributing to, but also going beyond, the variation in higher-order traits. Recent research in the field of personality nuances has dealt with the stability, heritability, and predictive validity of these smallest personality traits. In key studies, Mõttus and colleagues evaluated the consensual validity, differential continuity, and heritability of nuances, operationalized as the 240 items of the NEO-PI-R in a German (Mõttus et al., 2017) and a larger cross-cultural sample (Mõttus et al., 2018). Aggregating the results of both studies, the consensual validity (median $=.12$ ) and differential continuity of personality nuances over on average 12 years (median $=.24$ ) were demonstrated. In addition, they found significant associations of item variance with gender and age as well as predictive validity for items linked to specific interests (e.g., sports). In the same vein, Seeboth and Mõttus (2018) examined the incremental predictive validity of personality nuances over and above Big Five domains, operationalized with the 50-item International Personality Item Pool Neuroticism Extraversion and Openness inventory (IPIP NEO; Goldberg, 1999), for various relevant life outcomes (e.g., general health, body mass index). Although the total predictive advantage they found for item models vs. domain models was small in absolute terms $(1.3 \%$ on average), the relative increase in explained variance was substantial (with 30\%), despite only using 50 items (Seeboth \& Mõttus, 2018). The authors presumed that with more extensive 


\section{CROSS-CULTURAL DIFFERENCES IN PERSONALITY NUANCES}

item inventories the probability of yielding larger predictive validity is higher, which is an issue considered in the current study.

\section{The Present Study}

The cross-cultural personality literature suggests that personality trait domains and facets show considerable mean level differences across countries. However, the severe lack of measurement invariance or presence of DIF (Dong \& Dumas, 2020; Jankowsky et al., in press) suggests that a large proportion of item or nuance level differences are not accounted for by the overarching facet and trait domain levels. In this study, we examine the degree of cross-cultural variations at each level of the personality model hierarchy. More specifically, we investigate to what extent trait domains, facets, and items of the 300-item IPIP-NEO (Goldberg, 1999) can be used to predict the nationality of 9,110 participants from ten different countries. We used machine learning approaches to predict the nationality of participants using personality trait domains, facets, and items as predictors. In more detail, we applied pairwise logistic regressions to predict nationality and used cross-validation and elastic net regularization to ensure the robustness of the results (see also Mõttus \& Rozgonjuk, 2019; Seeboth \& Mõttus, 2018). We then compared the classification accuracy based on domains, facets and items to quantify the degree of cross-cultural differences at each level of the personality hierarchy. We also used the classification accuracy for each pairwise comparison of countries as an indicator of the degree of personality-relevant cultural differences between countries.

Based on findings supporting the notion that personality traits are geographically distributed (e.g., Allik et al., 2017; Allik \& McCrae, 2004; McCrae et al., 1998; McCrae \& Terracciano, 2005b; Schmitt et al., 2007), the severe lack of measurement invariance in crosscultural personality studies (Dong \& Dumas, 2020; Jankowsky et al., in press), and recent research on personality nuances’ relevance beyond trait domains and facets (e.g., Elleman et al., in press; Mõttus et al., 2018; Mõttus \& Rozgonjuk, 2019; Seeboth \& Mõttus, 2018), we 


\section{CROSS-CULTURAL DIFFERENCES IN PERSONALITY NUANCES}

expect that nuances are more predictive of country ${ }^{1}$ membership than facets, which in turn are more predictive than the trait domains. In other words, we expect systematic cross-cultural variance to be most substantively located at the level of specific thoughts, emotions, behavior, et cetera, which are not fully captured at the facet or domain level of personality.

\section{Method}

\section{Participants}

We used a subset of an openly available personality dataset of the IPIP-NEO 300 (Johnson, 2014; https://osf.io/tbmh5/), which was collected online (http://www.personal.psu.edu/ j5j/IPIP/). Out of the initial sample of 307,313 participants, who reported to come from all over the world (counting states as well as independent islands), we selected a subsample of 10 countries: Australia, Canada, India, Malaysia, New Zealand, the Philippines, Singapore, South Africa, the UK, and the USA. An important criterion in the selection was that English is an official language in these countries. As the personality questionnaire was administered in English, this was a way of reducing potential response bias due to a lack of language skills. Moreover, this selection provides a culturally diverse sample, comprising neighboring regions (e.g., the USA and Canada) as well as geographically widely remote countries (e.g., Singapore and the UK).

To account for possible biases in the comparison of personality scores due to age and gender differences across groups, we performed a propensity score matching of the countrysamples by the two covariates (Luellen et al., 2005). For that, Malaysian participants, who comprised the smallest sample $(N=911)$, were chosen as a reference group. Via one-to-one matching and the nearest neighbor method, the participants of each country were matched

\footnotetext{
${ }^{1}$ While the terms country and culture have often been used synonymously in the literature, both refer to different compositions of larger groups of people and the features that define them. Thereby, we consider "country" (or state/nation), in its most basic definition, to refer to the geographical localization of a people on the world map, whereas "culture" defines a broader concept of shared attributes, such as religion, traditions, customs and language, that define groups of individuals, going beyond national borders.
} 


\section{CROSS-CULTURAL DIFFERENCES IN PERSONALITY NUANCES}

with the participant most similar in age and sex in the Malaysian sample (for an overview of the matching procedures see Ho et al., 2007). This resulted in a mostly balanced composition of participants across countries with respect to age and gender (see Table 1). Consequently, the final total sample was composed of $N=9,110$ participants, of whom $42 \%(3,856)$ were male and 58\% $(5,254)$ female, with an average age of $M=23.5$ years $(S D=7.25)$, ranging from 12 to 61 years (the pre-matched data had initially provided an age range between 12 and 90). Matching was done with the MatchIt R-package (Ho et al., 2007b). About $0.4 \%$ of the responses were missing on the item level.

Table 1.

Descriptive Statistics of Participant Distribution after Propensity Score Matching

\begin{tabular}{lccccc}
\hline Country & $N$ & Female & Male & $M_{\text {Age }}$ & SD $D_{\text {Age }}$ \\
\hline & & & & & \\
Australia & 911 & 522 & 389 & 23.09 & 7.04 \\
Canada & 911 & 522 & 389 & 23.09 & 7.04 \\
India & 911 & 539 & 372 & 23.52 & 6.79 \\
Malaysia & 911 & 522 & 389 & 23.09 & 7.04 \\
New Zealand & 911 & 524 & 387 & 23.09 & 7.03 \\
Philippines & 911 & 555 & 356 & 23.29 & 7.41 \\
Singapore & 911 & 521 & 390 & 23.09 & 7.06 \\
South Africa & 911 & 505 & 406 & 26.72 & 8.16 \\
UK & 911 & 522 & 389 & 23.09 & 7.04 \\
USA & 911 & 522 & 389 & 23.09 & 7.04 \\
& & & & & \\
Total & 9,110 & 5,254 & 3,856 & 23.52 & 7.25 \\
\hline
\end{tabular}

\section{Measures}

Personality was measured with the English version of the IPIP-NEO 300 (Goldberg, 1999), a 300-item measure of the five factors and corresponding facets of personality (Costa and McCrae; 1992). Each Big Five factor is measured by six facets, which in turn are measured by ten items each. The IPIP-NEO 300 scales are balanced in item keying, thus reducing acquiescence bias. The full inventory can be found on the international personality 


\section{CROSS-CULTURAL DIFFERENCES IN PERSONALITY NUANCES}

item pool homepage (https://ipip.ori.org/). Participants indicated their agreement to short statements (e.g., “Worry about things”) on a 5-point Likert scale ( 1 = very inaccurate, 2 = moderately inaccurate, 3 = neither accurate nor inaccurate, $4=$ moderately accurate, and $5=$ very accurate; see supplementary material from Johnson, 2014).

\section{Statistical analysis}

To estimate the degree of cross-cultural differences at the trait nuance, facet, and domain level, we predicted the nationality of the participants. For this purpose, we applied multiple logistic regressions using the nuance (i.e., the 300 items), facet, and domain scores as predictors of the participants' country of residence. This was done for each pair of countries, totaling 45 comparisons, which allowed us to identify groups of similar and distinct countries in regard to the personality of the inhabitants. Countries were dummy-coded, so that the results of the logistic regression were an estimation of the probability of group membership, ranging from 0 (= participant certainly comes from the country coded with 0 ) to 1

(= participant certainly comes from the country coded with 1 ) with a cutoff of .50. Item-based models with hundereds of predictors tend to explain more variance than domain- or facetbased models for purely statistical reasons (see also Seeboth \& Mõttus, 2018). To account for this overfitting, we used cross-validation (Yarkoni \& Westfall, 2017) with $80 \%$ of the data ( $N$ $=7,288)$ to train the models, and the remaining $20 \%(N=1,822)$ to test model performance in an independent sample; the cross-validation was repeated three times. Domain and facet models were estimated with all predictors (i.e., 5 and 30, respectively). For the nuance models with all 300 items as predictors ${ }^{2}$, we applied elastic net regularization with the glmnet Rpackage (Friedman et al., 2010) to improve the robustness of the regression parameters. Regularized (or penalized) regression methods are designed to substantially reduce the (error)

\footnotetext{
${ }^{2}$ Because the regression-based machine learning approaches require complete data, we imputed missing values with respondents' mean values on the corresponding facet. We note that such an imputation procedure can lead to a bias of variance coefficients (e.g., Graham, 2009), but in the present case the number of imputed values was negligable.
} 


\section{CROSS-CULTURAL DIFFERENCES IN PERSONALITY NUANCES}

variance of regression coefficients at the expense of introducing slight bias to the estimated parameters (Helwig, 2017). Two forms of penalized regression that are often used to improve model accuracy are ridge regression (Hoerl \& Kennard, 1970) and the least absolute shrinkage and selection operator (LASSO) (Tibshirani, 1996). While LASSO regression uses a penalization method which combines a continuous shrinkage of the regression coefficients and an automatic variable selection to calculate sparser prediction models (Tibshirani, 1996, 2011), ridge regression uses a penalization procedure that shrinks the coefficients of correlated predictors to 0, but retains the total number of predictors (Hoerl \& Kennard, 1970). Accordingly, LASSO regression develops a more parsimonious and at the same time similarly as accurate model as ridge regression, which makes it a dominant method of training regression models preferred by researchers and statisticians (Zou \& Hastie, 2005). However, the LASSO tends to perform rather poorly when predictor variables are highly correlated (e.g., Friedman et al., 2010; Waldmann et al., 2013). In this case, LASSO usually selects only one predictor from the group of correlated variables and ignores the other predictors. Elastic Net regression, which was used in the subsequent analyses, offers a compromise between the ridge and the LASSO regression penalties by averaging the coefficients of strongly correlated predictors and including them in the model, at the same time providing a more parsimonious model by variable selection (Friedman et al., 2010; Zou \& Hastie, 2005). Elastic Net regression relies on two regularization parameters: $\lambda$ is the regularization parameter that represents the balance between model fitting and parameter shrinkage (Helwig, 2017). The goal of the parameter tuning is to find a $\lambda$ that provides an optimal balance while minimizing the mean square error term (MSE) (Helwig, 2017). The penalty parameter $\alpha$ regulates how much weight is given to either the LASSO or ridge regression. An $\alpha$ set to 0 is equivalent to ridge regression, while an $\alpha$ close to 1 performs similar to the LASSO (Waldmann et al., 2013). In our analysis, the optimal tuning parameters of $\lambda$ and $\alpha$ were obtained using three times 10-fold cross-validation on a variety of alpha levels within the training sample. The 


\section{CROSS-CULTURAL DIFFERENCES IN PERSONALITY NUANCES}

numbers of selected predictors in the item models varied with a range of 51 selected item predictors for the contrasting regression between Australia and New Zealand, and 260 selected item predictors for the contrasting regression between India and the USA. To foster transparency and reproducability we provide the matched data file and all syntax within the Open Science Framework (Soderberg, 2018) at

\section{https://osf.io/fwy5g/?view_only=26f8cb793f3448539ea25eb458ecd3c1.}

\section{Results}

To compute the discriminative accuracy of the logistic regressions, we calculated the “Area Under the ROC (receiver operating characteristic) Curve”. The AUC value gives the probability with which, out of two randomly drawn subjects from different countries, the subject coming from country 1 will receive a higher predictive score than the subject coming from country 0 (Fawcett, 2006). AUC values generally range from .50 (i.e., the model is as good as a random assignment of participants to countries) to 1.00 (i.e., the model perfectly discriminates between participants from different countries). Figure 1 shows the classification accuracy of all pairwise country comparisons stratified by the three personality levels and two datasets (i.e., training and validation sample) used. AUC values of the training and validation sample are close together, yielding a robust classification. That means, the elastic net regularization ensured that the 300-item models are not overfitted. However, one pairwise country comparison, namely Australia vs. New Zealand, was on chance level in the validation sample (see outlier in Figure 1). Consistent with our expectations, the discriminative abilities considerably increased from the Big Five models ( $M_{\mathrm{AUC}}$ in training/validation sample $=$ $.612 / .602)$ over the facet models $\left(M_{\mathrm{AUC}}\right.$ in training/validation sample $\left.=.753 / .729\right)$ to the item models $\left(M_{\mathrm{AUC}}\right.$ in training/validation sample $\left.=.946 / .886\right)$; these differences were robust and substantial $(p<.001)$. 
Figure 1

Classification Accuracy Across Personality Levels and Samples

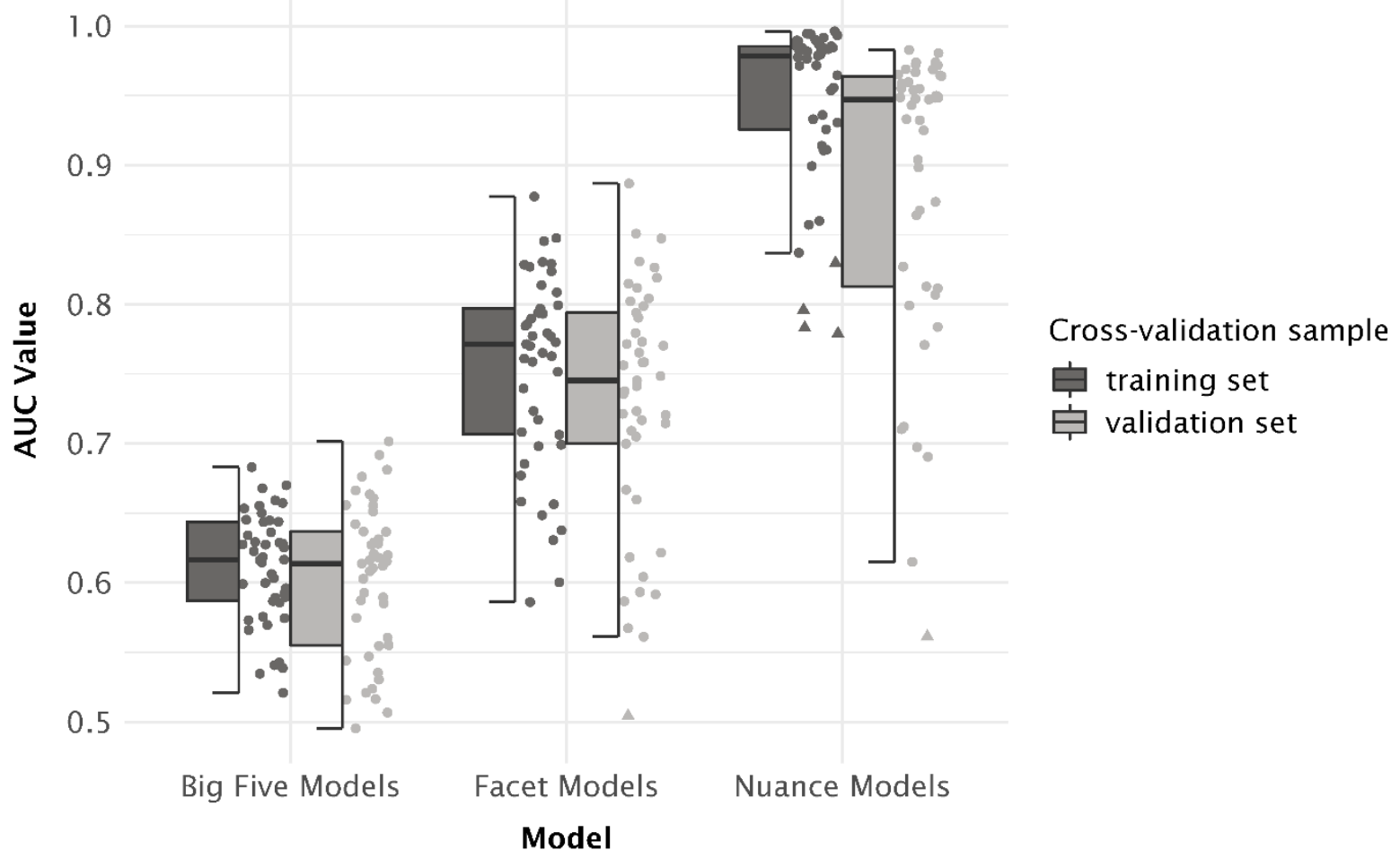

Note. The halved boxplot reflects the interquartile range, the solid line represents the median, and the whiskers 1.5 times the interquartile range. In addition, individual AUC values are displayed as jittered distribution on the right with outliers as triangles.

A high AUC value indicates that the personality differences between two countries are so substantial that we can accurately predict the country of residence just by comparing the personality trait levels between participants. Vice versa, a low AUC (i.e., close to .50) would suggest that participants from two different countries are similar in regard to their distribution of personality trait levels, and arguably personality-relevant cultural norms. We computed the opposite probability of the AUC (i.e., 1 - AUC) as an indicator of the similarity of personality levels across countries. Values close to .50 thus indicate that the personality of participants from different countries is equivalent, whereas a value of 0 indicates that the differences are so strong that the country of residence can be perfectly predicted based on the personality of each participant. Figure 2 shows this metric of shared personality across the three different levels for each pairwise country comparison in the validation sample. 
Figure 2

Similarity of Countries Based on Domain, Facet, and Nuance Models’ Performance in the Validation Set
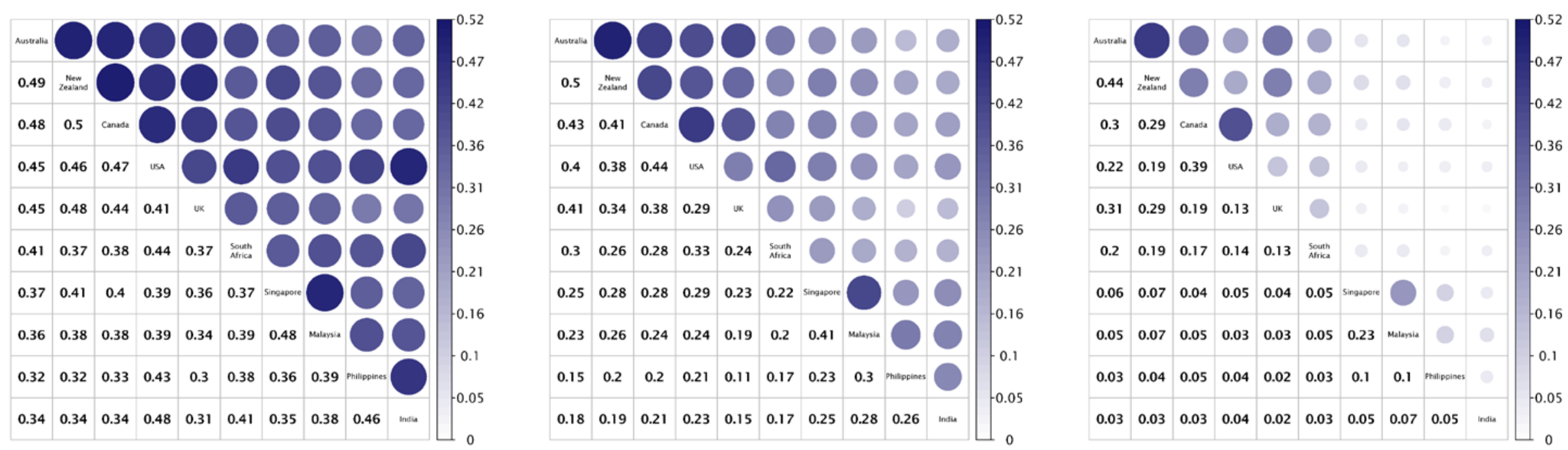

Note. Values represent the opposite probability of the AUC (=1-AUC) between two countries. Values close to 0 indicate large differences. 


\section{CROSS-CULTURAL DIFFERENCES IN PERSONALITY NUANCES}

At the trait domain level, similarities between all countries were relatively high, which seems to support the assumption of the universal applicability of personality (Allik \& McCrae, 2004; Heine \& Buchtel, 2009), including countries for which the empirical support for the Five-Factor structure was mixed (e.g., for Malaysia see McCrae \& Terracciano, 2005a). However, at the facet level a more diverse picture emerges: Australia, New Zealand, Canada, the USA, and the UK form a cluster of countries with a similar facet level structure; these countries represent the Western, educated, industrialized, rich, and democratic (WEIRD; Henrich et al., 2010) countries used in this study. Also the participants from South Africa were attached to the cluster of WEIRD countries, which may be caused by the prominent position in economic and historic aspects. Across the Asian countries, differences were much more apparent; the only exception being Malaysia and Singapore with considerable facet level similarities, arguably because of the geographical neighborhood of these countries as well as their strong historical, political, and economic ties. Table 2 shows examples of the most important facet and nuance-level variables in the distinction of some selected countries (Greenwell et al., 2018). India and the Philippines, for instance, differed considerably in the Openness facets Emotionality, Liberalism, and Artistic Interest, with India scoring higher in the first two and lower in the latter. Artistic Interest, and Liberalism also seem to be most pred2

ictive in distinguishing between the Philippines and a WEIRD country such as the UK, for example.

At the nuance level, personality-related differences between countries were much stronger than at the facet level. Participants from New Zealand and Australia (AUC $=.57$ ) and Canada and the USA (AUC $=.62$ ) were still very similar in regard to their personality at the nuance level. Additionally, Australia and Canada (AUC = .68) showed high nuance-specific similarities. Surprisingly, differences between the UK and the USA or Canada became much more apparent at the nuance level. Participants from the USA/Canada showed higher 


\section{CROSS-CULTURAL DIFFERENCES IN PERSONALITY NUANCES}

agreement than the UK participants on specific items such as "Believe laws should be strictly enforced” (Openness: Liberalism), and “Excel in what I do” (Conscientiousness: SelfEfficacy) (see Table 2). We found the largest differences in personality nuances between the cluster of WEIRD and Asian (Malaysia, Singapore, the Philippines, and India) countries. Most notably, participants from the Philippines, which was the only country without historical ties to the British Empire, and India, which was the first of the Asian countries to gain its independence from the British Empire, were most distinct from participants of the other countries on all personality trait levels. Some of the items most responsible for these nuancelevel differences were "Believe laws should be strictly enforced” (Openess:Liberalism), and "Believe that I am better than others.” (Agreeableness: Modesty), in which the U.S. sample, for instance, endorsed more readily than the Filipino sample. Please note that we singled out specific items here, but that the estimates of variable importance are not very stable and the order might change across various iterations. 
CROSS-CULTURAL DIFFERENCES IN PERSONALITY NUANCES

Table 2.

Relative Variable Importance of Predictors in Facet and Nuance Models

\begin{tabular}{|c|c|c|c|c|c|c|c|c|}
\hline \multirow{2}{*}{$\begin{array}{c}\text { Facet models } \\
\text { Facet } \\
\end{array}$} & \multirow[b]{2}{*}{ Domain } & \multicolumn{5}{|c|}{ Nuance models } & \multirow[b]{2}{*}{$\begin{array}{c}\text { Relative } \\
\text { Importance }\end{array}$} & \multirow[b]{2}{*}{$\begin{array}{l}\text { Predictec } \\
\text { Direction }\end{array}$} \\
\hline & & $\begin{array}{c}\text { Relative } \\
\text { Importance }\end{array}$ & $\begin{array}{l}\text { Predicted } \\
\text { Direction } \\
\end{array}$ & Item No. & Item Description & Domain/Facet & & \\
\hline \multicolumn{9}{|c|}{ UK vs. USA } \\
\hline Artistic Interest & $\mathrm{O}$ & 9.19 & USA & 35 & Excel in what I do. & C: Self-Efficacy & 0.37 & USA \\
\hline Liberalism & $\mathrm{O}$ & 6.33 & UK & 178 & Believe that too much tax money goes to support artists. & O: Liberalism & 0.35 & USA \\
\hline Intellect & $\mathrm{O}$ & 4.90 & UK & 208 & Believe laws should be strictly enforced. & O: Liberalism & 0.32 & USA \\
\hline Self-Efficacy & $\mathrm{C}$ & 3.26 & UK & 83 & Have a rich vocabulary. & O: Intellect & -0.31 & UK \\
\hline Immoderation & $\mathrm{N}$ & 3.01 & USA & 58 & Believe that there is no absolute right or wrong. & O: Liberalism & -0.31 & UK \\
\hline Activity-Level & $\mathrm{E}$ & 2.77 & UK & 298 & Like to stand during the national anthem. & O: Liberalism & -0.65 & UK \\
\hline \multicolumn{9}{|c|}{ Philippines vs. USA } \\
\hline Trust & A & 9.16 & PHL & 208 & Believe laws should be strictly enforced. & O: Liberalism & 0.50 & USA \\
\hline Adventurousness & $\mathrm{O}$ & 7.68 & PHL & 144 & Believe that I am better than others. & A: Modesty & 0.34 & USA \\
\hline Cooperation & A & 5.80 & PHL & 236 & Know how to cope. & N: Vulnerability & 0.27 & USA \\
\hline Gregariousness & $\mathrm{E}$ & 4.86 & PHL & 167 & Like to take it easy. & E: Activity-Level & -0.23 & PHL \\
\hline Dutifulness & $\mathrm{C}$ & 4.81 & USA & 87 & Express childlike joy. & E: Cheerfulness & -0.24 & PHL \\
\hline Orderliness & $\mathrm{C}$ & 4.74 & USA & 234 & Know the answers to many questions. & A: Modesty & -0.30 & PHL \\
\hline \multicolumn{9}{|c|}{ Philippines vs. UK } \\
\hline Artistic Interest & $\mathrm{O}$ & 10.13 & PHL & 298 & Like to stand during the national anthem. & O: Liberalism & 0.35 & UK \\
\hline Trust & A & 8.65 & PHL & 169 & Love a good fight. & A: Cooperation & 0.28 & UK \\
\hline Liberalism & $\mathrm{O}$ & 7.58 & UK & 149 & Am not interested in other people's problems. & A: Sympathy & 0.27 & UK \\
\hline Gregariousness & $\mathrm{E}$ & 6.92 & UK & 234 & Know the answers to many questions. & A: Modesty & -0.22 & PHL \\
\hline Activity-Level & $\mathrm{E}$ & 5.80 & UK & 278 & Do not enjoy watching dance performances. & O: Artistic Interest & -0.25 & PHL \\
\hline Intellect & $\mathrm{O}$ & 5.33 & UK & 127 & Love surprise parties. & E: Gregariousness & -0.25 & PHL \\
\hline \multicolumn{9}{|c|}{ India vs. Philippines } \\
\hline Immoderation & $\mathrm{N}$ & 7.05 & PHL & 205 & Waste my time. & C: Self-Discipline & 0.37 & PHL \\
\hline Emotionality & $\mathrm{O}$ & 5.42 & IND & 275 & Don't see the consequences of things. & C: Self-Efficacy & 0.36 & PHL \\
\hline Depression & $\mathrm{N}$ & 4.83 & IND & 298 & Like to stand during the national anthem. & O: Liberalism & 0.34 & PHL \\
\hline Modesty & A & 4.73 & PHL & 98 & Love flowers. & O: Artistic Interest & -0.33 & IND \\
\hline Artistic Interest & $\mathrm{O}$ & 4.56 & PHL & 161 & Feel desperate. & N: Depression & -0.36 & IND \\
\hline Liberalism & $\mathrm{O}$ & 4.39 & IND & 279 & Obstruct others' plans. & A: Morality & -0.45 & IND \\
\hline
\end{tabular}

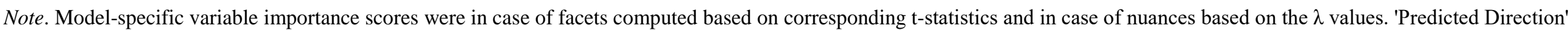
indicates which country shows stronger agreement on the facet/item. PHL = Philippines, IND = India, AUS = Australia. 


\section{CROSS-CULTURAL DIFFERENCES IN PERSONALITY NUANCES}

\section{Discussion}

Attempts to explain differences in personality traits between countries have so far mostly focused on the trait domain, and sometimes the facet level of personality. However, recent studies demonstrated convincingly that individual personality test items contain substantial inter-individual personality differences that are not accounted for by the trait domain and facet level (McCrae, 2015; Mõttus et al., 2017, 2018; Seeboth \& Mõttus, 2018). The severe issue of differential item functioning in cross-cultural personality studies (e.g., Dong \& Dumas, 2020) indicates that this nuance-level variance is either affected or expressed by cultural differences. In the current study, we examined and quantified cross-cultural differences on the trait domain, facet, and nuance level of personality. Moreover, we identified clusters of countries that were more similar vs. dissimilar on the different levels of the hierarchy. Using the trait domains, the Big Five, as predictors of country of residence, only about $60 \%$ of participants could be assigned to their respective country of residence in the validation sample correctly, which is only slightly better than any assignment by chance. Using the facet level scores, we correctly identified the country of residence in $75 \%$ of the cases. Based on the personality items or nuances, $89 \%$ of participants were assigned correctly. This pattern indicates that cross-cultural differences are much more apparent at the lower levels of personality (i.e., facets and most importantly nuances) than at the trait domain level. As such, it is not surprising that studies examining the differences at the trait domain level only found small differences (Allik et al., 2017; Allik \& McCrae, 2004; McCrae et al., 1998; McCrae \& Terracciano, 2005b; Schmitt et al., 2007), whereas studying cross-cultural differences at the narrower trait levels offers a more informative picture of differences and similarities between countries.

Culture is a complex construct which is influenced by geographical, sociological, historical, linguistic, religious, and psychological factors, so that no academic discipline can claim to explain cultural differences on its own. Accordingly, any explanatory attempts must 


\section{CROSS-CULTURAL DIFFERENCES IN PERSONALITY NUANCES}

also be multifaceted. Consistent with findings from Allik and McCrae (2004) geographically proximate countries such as the USA and Canada, Australia and New Zealand, and Malaysia and Singapore are more similar than geographically distant countries. Accordingly, immigration movements may have promoted the exchange and rapprochement between cultures, at least in times before digitalization and enhanced mobility. From a sociological perspective, cultural differences in personality have often been linked to Hofstede's cultural dimensions (Hofstede, 1980, 2001) such as Individualism, Power Distance (the "degree to which the less powerful members of a society accept and expect that power is distributed unequally”, Hofstede, 2020) or Indulgence (the degree, to which “a society ... allows relatively free gratification of basic and natural human drives related to enjoying life and having fun”, Hofstede, 2020). However, the concept of cultural dimensions only moves the problem into new container terms on a societal level. From a historic perspective, it has been rightly noted that special attention should be placed on the "historical forces that shape these differences” (Okazaki et al., 2008), a circumstance to which we allude to in the title of the present paper. Put differently, similarities and differences in cultural norms are not fixed but prone to change over time. With regard to the possible explanations, we do not want to speculate and limit the discussion to the measurement aspect.

Cultural differences can be studied from two opposing or complementing viewpoints (see also Church et al., 2011). Cross-cultural variability is seen as nuisance that threatens comparability of certain results across countries. According to a recent review, no crosscultural personality study so far has achieved scalar measurement invariance (Dong \& Dumas, 2020), which is required for an unbiased comparison of the factor mean levels across countries. Some studies tried to tackle the measurement invariance issue by systematically eliminating items that exhibited a large amount of DIF (Jankowsky et al., in press; Olaru \& Danner, in press). But suppressing the existent variability also comes at the expense of the breadth of the measurement, which is also known as bandwidth-fidelity dilemma (Cronbach 


\section{CROSS-CULTURAL DIFFERENCES IN PERSONALITY NUANCES}

\& Gleser, 1957). Thus, non-invariant items might not be a mere nuisance, but of special interest when it comes to cultural differences, as they might indicate basic differences in attitudes, thoughts, emotions, motives, and behavior. The large differences in classification accuracy between nuances and the other levels of the personality hierarchy corroborate this notion: Cross-cultural differences manifest on the item level of personality beyond the facets and trait domains, which is astonishing since the items used in the IPIP-NEO 300 (Goldberg, 1999) are very general in their wording, in particular in comparison to the original NEO-PI-R (Costa \& McCrae, 1992), which contains highly specific formulations that can cause such issues of DIF (e.g., "I love the excitement of roller coasters”, see Church et al., 2011; Olaru et al., 2019). Notwithstanding the general, plain language of the measure and the fact that we restricted our examination to countries with English as an official language, we found substantial differences between countries on the item level which also question the universality of personality on all levels of the hierarchy. Although the item inventory used in this study is already one of the more comprehensive inventories utilized in personality research and applied areas, it is unlikely to exhaust the actual amount of personality nuances that can be found in the Big Five framework (Mõttus \& Rozgonjuk, 2019). Another limitation of the present study is the the analyses are based on an online sample that is not representative of the general population of the countries in terms of age (with only $14 \%$ of the sample older than 30 years) and and presumably other (not documented) characteristics. Due to the young age of participants and recruitement via internet, the majority of the sample presumably comprises of college students and young professionals, which may represent a more Westernized socioeconomic elite (Allik \& McCrae, 2004). Second, despite selecting countries with English as one of the official languages, the English language proficiency of participants outside of the United States, United Kingdom, Canada, Australia, and New Zealand will inevitably be lower than of participants from the aforementioned countries. These language differences might have introduced a method bias and artificially increased the differences to 


\section{CROSS-CULTURAL DIFFERENCES IN PERSONALITY NUANCES}

the non-WEIRD countries used in this study. Third, regional differences in personality can also be observed within countries on state level (see Elleman et al., in press). Therefore, future research should consider the analysis of a wider spectrum of culturally diverse units, which might also include lower-level geographical units within a country.

\section{Conclusion}

In this study, we examined cross-cultural differences in the trait domain, facet, and nuance level of personality by predicting the country of residence based on the responses of 9,110 age- and gender-matched participants from the US, UK, Canada, Australia, New Zealand, South Africa, Malaysia, Singapore, India, and the Philippines on the IPIP-NEO 300. The results suggest that only a small proportion of cross-cultural personality differences manifest at the broad trait domain level, while essential information on cross-cultural differences are located at the facet and, most prominently, nuance level. These findings also partially explain the lack of measurement invariance commonly reported in cross-cultural personality studies. When looking at similarities between countries in respect to personality nuances, we found a cluster of the WEIRD countries, and high similarities between Malaysia and Singapore. In contrast, all other countries included in this study showed considerable personality differences to each other. 


\section{References}

Allik, J., Church, A. T., Ortiz, F. A., Rossier, J., Hřebíčková, M., de Fruyt, F., Realo, A., \& McCrae, R. R. (2017). Mean profiles of the NEO Personality Inventory. Journal of Cross-Cultural Psychology, 48(3), 402-420. https://doi.org/10.1177/0022022117692100

Allik, J., \& McCrae, R. R. (2004). Toward a geography of personality traits: Patterns of profiles across 36 cultures. Journal of Cross-Cultural Psychology, 35(1), 13-28. https://doi.org/10.1177/0022022103260382

Baer, R. A., Samuel, D. B., \& Lykins, E. L. B. (2011). Differential item functioning on the Five Facet Mindfulness Questionnaire is minimal in demographically matched meditators and nonmeditators. Assessment, 18(1), 3-10. https://doi.org/10.1177/1073191110392498

Borsboom, D. (2006). The attack of the psychometricians. Psychometrika, 71(3), 425-440. https://doi.org/10.1007/s11336-006-1447-6

Church, A. T., Alvarez, J. M., Mai, N. T. Q., French, B. F., Katigbak, M. S., \& Ortiz, F. A. (2011). Are cross-cultural comparisons of personality profiles meaningful? Differential item and facet functioning in the Revised NEO Personality Inventory. Journal of Personality and Social Psychology, 101(5), 1068-1089. https://doi.org/10.1037/a0025290

Clauser, B. E., \& Mazor, K. M. (1998). Using statistical procedures to identify differentially functioning test items. Educational Measurement: Issues and Practice, 17(1), 31-44. https://doi.org/10.1111/j.1745-3992.1998.tb00619.x

Costa, P. T., \& McCrae, R. R. (1992). Revised NEO Personality Inventory (NEO-PI-R) and NEO Five-Factor (NEO-FFI) inventory professional manual. Odessa, Fl: PAR.

Cronbach, L. J., \& Gleser, G. C. (1957). Psychological tests and personnel decisions. University of Illinois Press. 


\section{CROSS-CULTURAL DIFFERENCES IN PERSONALITY NUANCES}

Davidov, E., Meuleman, B., Cieciuch, J., Schmidt, P., \& Billiet, J. (2014). Measurement equivalence in cross-national research. Annual Review of Sociology, 40(1), 55-75. https://doi.org/10.1146/annurev-soc-071913-043137

Dong, Y., \& Dumas, D. (2020). Are personality measures valid for different populations? A systematic review of measurement invariance across cultures, gender, and age. Personality and Individual Differences, 160, 109956.

Eigenhuis, A., Kamphuis, J. H., \& Noordhof, A. (2013). Development and validation of the Dutch Brief Form of the Multidimensional Personality Questionnaire (MPQ-BF-NL). Assessment, 20(5), 565-575. https://doi.org/10.1177/1073191112444920

Eigenhuis, A., Kamphuis, J. H., \& Noordhof, A. (2015). Personality differences between the United States and the Netherlands: The influence of violations of measurement invariance. Journal of Cross-Cultural Psychology, 46(4), 549-564. https://doi.org/10.1177/0022022115570671

Elleman, L. G., Condon, D. M., Holtzman, N. S., Allen, V. R., \& Revelle, W. (in press). Smaller is better: Associations between personality and demographics are improved by examining narrower traits and regions [Preprint]. PsyArXiv. https://doi.org/10.31234/osf.io/dpmg2

Epskamp, S. (2017). Network psychometrics [University of Amsterdam]. https://dare.uva.nl/search?identifier=a76273c6-6abc-4cc7-a2e93b5f1ae3c29e

Fawcett, T. (2006). An introduction to ROC analysis. Pattern Recognition Letters, 27(8), 861-874. https://doi.org/10.1016/j.patrec.2005.10.010

Friedman, J., Hastie, T., \& Tibshirani, R. (2010). Regularization Paths for Generalized Linear Models via Coordinate Descent. Journal of Statistical Software, 33(1). https://doi.org/10.18637/jss.v033.i01 


\section{CROSS-CULTURAL DIFFERENCES IN PERSONALITY NUANCES}

Goldberg, L. R. (1990). An alternative “description of personality”: The Big-Five factor structure. Journal of Personality and Social Psychology, 59(6), 1216.

Goldberg, L. R. (1999). A broad-bandwidth, public domain, personality inventory measuring the lower-level facets of several Five-Factor models. Personality Psychology in Europe, 7(1), 7-28.

Graham, J. W. (2009). Missing data analysis: Making it work in the real world. Annual Review of Psychology, 60, 549-576.

Greenwell, B. M., Boehmke, B. C., \& McCarthy, A. J. (2018). A simple and effective modelbased variable importance measure. ArXiv:1805.04755 [Cs, Stat]. http://arxiv.org/abs/1805.04755

Heine, S. J., \& Buchtel, E. E. (2009). Personality: The universal and the culturally specific. Annual Review of Psychology, 60(1), 369-394. https://doi.org/10.1146/annurev.psych.60.110707.163655

Helwig, N. E. (2017). Adding bias to reduce variance in psychological results: A tutorial on penalized regression. The Quantitative Methods for Psychology, 13(1), 1-19. https://doi.org/10.20982/tqmp.13.1.p001

Henrich, J., Heine, S. J., \& Norenzayan, A. (2010). The weirdest people in the world? Behavioral and Brain Sciences, 33(2-3), 61-83. https://doi.org/10.1017/S0140525X0999152X

Ho, D. E., Imai, K., King, G., \& Stuart, E. A. (2007). Matching as nonparametric preprocessing for reducing model dependence in parametric causal inference. Political Analysis, 15(3), 199-236. https://doi.org/10.1093/pan/mpl013

Ho, D. E., Imai, K., King, G., \& Stuart, E. A. (2011). MatchIt: Nonparametric preprocessing for parametric causal inference. Journal of Statistical Software, 42(8). https://doi.org/10.18637/jss.v042.i08 


\section{CROSS-CULTURAL DIFFERENCES IN PERSONALITY NUANCES}

Hoerl, A. E., \& Kennard, R. W. (1970). Ridge regression: Biased estimation for nonorthogonal problems. Technometrics, 12(1), 55-67. https://doi.org/10.1080/00401706.1970.10488634

Hofstede, G. (1980). Culture's consequences: International differences in work-related values. Sage Publications.

Hofstede, G. (2001). Culture's consequences: Comparing values, behaviors, institutions and organizations across nations. Sage Publications.

Hofstee, W. K., De Raad, B., \& Goldberg, L. R. (1992). Integration of the big five and circumplex approaches to trait structure. Journal of personality and social psychology, 63(1), 146. https://doi.org/10.1037/0022-3514.63.1.146

Huang, C. D., Church, A. T., \& Katigbak, M. S. (1997). Identifying cultural differences in items and traits: Differential item functioning in the NEO Personality Inventory. Journal of Cross-Cultural Psychology, 28(2), 192-218. https://doi.org/10.1177/0022022197282004

Jankowsky, K., Olaru, G., \& Schroeders, U. (in press). Compiling measurement invariant short scales in cross-cultural personality assessment using Ant Colony Optimization. European Journal of Personality.

Johnson, J. A. (2014). Measuring thirty facets of the Five Factor Model with a 120-item public domain inventory: Development of the IPIP-NEO-120. Journal of Research in Personality, 51, 78-89. https://doi.org/10.1016/j.jrp.2014.05.003

Johnson, T., Kulesa, P., Cho, Y. I., \& Shavitt, S. (2005). The relation between culture and response styles: Evidence from 19 countries. Journal of Cross-Cultural Psychology, 36(2), 264-277.

Kajonius, P., \& Mac Giolla, E. (2017). Personality traits across countries: Support for similarities rather than differences. PLoS ONE, 12(6). https://doi.org/10.1371/journal.pone.0179646 


\section{CROSS-CULTURAL DIFFERENCES IN PERSONALITY NUANCES}

Lee, K., \& Ashton, M. C. (2004). Psychometric properties of the HEXACO personality inventory. Multivariate Behavioral Research, 39(2), 329-358.

Luellen, J. K., Shadish, W. R., \& Clark, M. H. (2005). Propensity scores: An introduction and experimental test. Evaluation Review, 29(6), 530-558.

https://doi.org/10.1177/0193841X05275596

Marsh, H. W., Lüdtke, O., Muthén, B., Asparouhov, T., Morin, A. J. S., Trautwein, U., \& Nagengast, B. (2010). A new look at the big five factor structure through exploratory structural equation modeling. Psychological Assessment, 22(3), 471-491. https://doi.org/10.1037/a0019227

McCrae, R. R. (2015). A more nuanced view of reliability: Specificity in the trait hierarchy. Personality and Social Psychology Review, 19(2), 97-112. https://doi.org/10.1177/1088868314541857

McCrae, R. R., \& Costa, P. T. (1985). The NEO Personality Inventory manual. Psychological Assessment Resources, Odessa.

McCrae, R. R., Costa, P. T., Del Pilar, G. H., Rolland, J.-P., \& Parker, W. D. (1998). Crosscultural assessment of the Five-Factor Model: The Revised NEO Personality Inventory. Journal of Cross-Cultural Psychology, 29(1), 171-188. https://doi.org/10.1177/0022022198291009

McCrae, R. R., \& Terracciano, A. (2005a). Universal features of personality traits from the observer's perspective: Data from 50 cultures. Journal of Personality and Social Psychology, 88(3), 547-561. https://doi.org/10.1037/0022-3514.88.3.547

McCrae, R. R., \& Terracciano, A. (2005b). Personality profiles of cultures: Aggregate personality traits. Journal of Personality and Social Psychology, 89(3), 407-425. https://doi.org/10.1037/0022-3514.89.3.407

Millsap, R. E. (2011). Statistical approaches to measurement invariance. Routledge. 


\section{CROSS-CULTURAL DIFFERENCES IN PERSONALITY NUANCES}

Mõttus, R., Kandler, C., Bleidorn, W., Riemann, R., \& McCrae, R. R. (2017). Personality traits below facets: The consensual validity, longitudinal stability, heritability, and utility of personality nuances. Journal of Personality and Social Psychology, 112(3), 474-490. https://doi.org/10.1037/pspp0000100

Mõttus, R., McCrae, R. R., Allik, J., \& Realo, A. (2014). Cross-rater agreement on common and specific variance of personality scales and items. Journal of Research in Personality, 52, 47-54. https://doi.org/10.1016/j.jrp.2014.07.005

Mõttus, R., \& Rozgonjuk, D. (2019). Development is in the details: Age differences in the Big Five domains, facets, and nuances. Journal of Personality and Social Psychology. https://doi.org/10.1037/pspp0000276

Mõttus, R., Sinick, J., Terracciano, A., Hřebíčková, M., Kandler, C., Ando, J., Mortensen, E. L., Colodro-Conde, L., \& Jang, K. L. (2018). Personality characteristics below facets: A replication and meta-analysis of cross-rater agreement, rank-order stability, heritability, and utility of personality nuances. Journal of Personality and Social Psychology, 117(4), 1-16. https://doi.org/10.1037/pspp0000202

Okazaki, S., David, E. J. R., \& Abelmann, N. (2008). Colonialism and psychology of culture. Social and Personality Psychology Compass, 2(1), 90-106.

\section{https://doi.org/10.1111/j.1751-9004.2007.00046.x}

Olaru, G., \& Danner, D. (in press). Developing cross-cultural personality short scales with metaheuristic item selection procedures. Assessment.

Olaru, G., Schroeders, U., Wilhelm, O., \& Ostendorf, F. (2019). ‘Grandpa, do you like roller coasters?’: Identifying age-appropriate personality indicators. European Journal of Personality, 33(3), 264-278.

Rammstedt, B., Danner, D., \& Bosnjak, M. (2017). Acquiescence response styles: A multilevel model explaining individual-level and country-level differences. 


\section{CROSS-CULTURAL DIFFERENCES IN PERSONALITY NUANCES}

Personality and Individual Differences, 107, 190-194.

https://doi.org/10.1016/j.paid.2016.11.038

R Core Team (2019). R: A language and environment for statistical computing. $R$ Foundation for Statistical Computing, Vienna, Austria. https://www.R-project.org/.

Rollock, D., \& Lui, P. P. (2016). Measurement Invariance and the Five-Factor Model of Personality: Asian International and Euro American Cultural Groups. Assessment, 23(5), 571-587. https://doi.org/10.1177/1073191115590854

Safdar, S., Friedlmeier, W., Matsumoto, D., Yoo, S. H., Kwantes, C. T., Kakai, H., \& Shigemasu, E. (2009). Variations of emotional display rules within and across cultures: A comparison between Canada, USA, and Japan. Canadian Journal of Behavioural Science/Revue Canadienne Des Sciences Du Comportement, 41(1), 1-10. https://doi.org/10.1037/a0014387

Schmitt, D. P., Allik, J., McCrae, R. R., \& Benet-Martínez, V. (2007). The geographic distribution of Big Five personality traits: Patterns and profiles of human selfdescription across 56 nations. Journal of Cross-Cultural Psychology, 38(2), 173-212. https://doi.org/10.1177/0022022106297299

Seeboth, A., \& Mõttus, R. (2018). Successful explanations start with accurate descriptions: Questionnaire items as personality markers for more accurate predictions: Items as personality markers. European Journal of Personality, 32(3), 186-201.

\section{https://doi.org/10.1002/per.2147}

Soderberg, C. K. (2018). Using OSF to share data: A step-by-step guide. Advances in Methods and Practices in Psychological Science, 1(1), 115-120. https://doi.org/10.1177/2515245918757689

Soto, C. J., \& John, O. P. (2017). The next Big Five Inventory (BFI-2): Developing and assessing a hierarchical model with 15 facets to enhance bandwidth, fidelity, and 


\section{CROSS-CULTURAL DIFFERENCES IN PERSONALITY NUANCES}

predictive power. Journal of Personality and Social Psychology, 113(1), 117-143. https://doi.org/0.1037/pspp0000096

Tibshirani, R. (1996). Regression shrinkage and selection via the lasso. Journal of the Royal Statistical Society: Series B (Methodological), 58(1), 267-288.

Tibshirani, R. (2011). Regression shrinkage and selection via the lasso: A retrospective. Journal of the Royal Statistical Society: Series B (Statistical Methodology), 73(3), 273-282. https://doi.org/10.1111/j.1467-9868.2011.00771.x

van de Vijver, F., \& Tanzer, N. K. (2004). Bias and equivalence in cross-cultural assessment: An overview. European Review of Applied Psychology, 54(2), 119-135. https://doi.org/10.1016/j.erap.2003.12.004

Waldmann, P., Mészáros, G., Gredler, B., Fuerst, C., \& Sölkner, J. (2013). Evaluation of the lasso and the elastic net in genome-wide association studies. Frontiers in Genetics, 4. https://doi.org/10.3389/fgene.2013.00270

Wong, N., Rindfleisch, A., \& Burroughs, J. E. (2003). Do reverse-worded items confound measures in cross-cultural consumer research? The case of the Material Values Scale. Journal of Consumer Research, 30(1), 72-91. https://doi.org/10.1086/374697

Yarkoni, T., \& Westfall, J. (2017). Choosing prediction over explanation in psychology: Lessons from machine learning. Perspectives on Psychological Science, 12(6), 1100_ 1122. https://doi.org/10.1177/1745691617693393

Zou, H., \& Hastie, T. (2005). Regularization and variable selection via the elastic net. Journal of the Royal Statistical Society: Series B (Statistical Methodology), 67(2), 301-320. https://doi.org/10.1111/j.1467-9868.2005.00503.x 